\title{
GRUPOS DIEDRAIS: \\ uma proposta concreta para uma apresentação inicial da Álgebra Abstrata para licenciandos em Matemática
}

\author{
DIHEDRAL GROUPS: \\ A CONCRETE PROPOSAL FOR AN INITIAL PRESENTATION OF \\ ABSTRACT ALGEBRA FOR GRADUATES IN MATHEMATICS
}

\author{
Andreza Thalia Menezes Monteiro* \\ Luiz Felipe Costa Mesquita Moura** \\ Rubens Vilhena Fonseca***
}

\section{Resumo}

Este artigo traz uma sugestão para o ensino/aprendizagem de tópicos específicos, referentes à Teoria dos Grupos nos cursos de Licenciatura em Matemática. A problemática consiste, inicialmente, no fato de que o tópico Grupos é considerado um assunto central a ser estudado nos cursos de licenciatura em Matemática em quase todas as universidades. As razões para essa centralidade podem variar consideravelmente, mas o consenso é que tempo e esforço devem ser dedicados ao seu ensino e aprendizado. Por outro lado, os estudantes têm sérias dificuldades cognitivas e afetivas com álgebra abstrata, ou seja, eles têm dificuldades em se tornar competentes e, mesmo entre os que obtêm sucesso, muitos não conseguem ver o objetivo em estudar tal assunto. Este artigo é uma tentativa de abordar uma pequena parte dessa problemática do ensino e aprendizagem da Álgebra Abstrata, com base na experiência acumulada por anos de pesquisa e desenvolvimento na apresentação do assunto teoria de grupos para os alunos de Licenciatura em Matemática da Universidade do Estado do Pará (UEPA). Espera-se que a abordagem apresentada possa servir como uma base para novas propostas e sugestões adicionais que possam aperfeiçoar o que aqui se sugere. Ao considerar as rotações e translações de movimentos rígidos nos polígonos regulares, em particular no triângulo equilátero e no quadrado, para deduzir os conceitos iniciais de grupos, este artigo pretende ser um recurso útil e animado para professores da disciplina Álgebra Abstrata e aos licenciandos.

Palavras-chave: Álgebra. Grupos. Licenciatura. Ensino e aprendizagem de álgebra. Abstrato e concreto.

\begin{abstract}
\footnotetext{
* Universidade do Estado do Para - e-mail: andrezathaliamm@gmail.com

*** Universidade do Estado do Pará - e-mail: luixfelipw@gmail.com

**** Universidade do Estado do Pará - e-mail: rubens.vilhena@uepa.br
}

This article provides a suggestion for the teaching/learning of specific topics, referring to Group Theory in mathematics degree courses. The problem consists, initially, in the fact that the topic Groups is considered a central subject to be studied in undergraduate courses in Mathematics in almost all universities. The reasons for this centrality may vary considerably, but the consensus is that time and effort should be devoted to their teaching and learning. On the other hand, students 
Grupos diedrais: uma proposta concreta para uma apresentação inicial da Álgebra Abstrata para licenciandos em Matemática

have serious cognitive and affective difficulties with abstract algebra, that is, they have difficulties in becoming competent and, even among those who succeed, many cannot see the goal in studying such a subject. This article is an attempt to embroider a small part of this problem of teaching and learning of Abstract Algebra, based on the experience accumulated by years of research and development in the presentation of the subject theory of groups for students of Degree in Mathematics of the Universidade do Estado do Pará (UEPA). The approach presented is expected to serve as a basis for further proposals and suggestions that can improve what is sucked here. When considering the rotations and translations of rigid movements in regular polygons, in the equilateral triangle and in the square, to deduce the initial concepts of groups, this article aims to be a useful and animated resource for teachers of the discipline Abstract Algebra and the licensors.

Key words: Algebra. Groups. Degree. Teaching and learning algebra. Abstract and concrete.

\section{Introdução}

A palavra álgebra tornou-se generalizada em meados do século XVI e deriva do árabe "Al Jabr" (ou Al Jebr), como foi usado pelo matemático Muhammad ibn Musa al-Khwarizmi no título de seu famoso tratado sobre equações "Kitab al-Jabr w'al-Muqabala" ('Regras de Reintegração e redução' ou 'Restauração e Equilíbrio') 1. Assim, a etimologia da palavra evoca as principais regras para resolver equações.

A palavra álgebra, hoje, indica diversas áreas dentro da Matemática, como a álgebra da escola ("elementar"), Álgebra Linear e Álgebra Abstrata.

Ao pesquisarmos as mais diferentes definições para a palavra álgebra, elas sugerem uma série de objetivos que, devido ao nosso tema principal, não serão discutidos neste artigo:

- Expressar generalizações;

- Estabelecer relações;

- Resolver problemas;

- Explorar propriedades;

- Provar teoremas;

- Calcular.

\footnotetext{
${ }^{1}$ Online Etymology Dictionary, 2001-2015
} 
Grupos diedrais: uma proposta concreta para uma apresentação inicial da Álgebra Abstrata para licenciandos em Matemática

Com respeito à álgebra abstrata, o que é que realmente fazemos? Quais são as ações físicas e mentais em que nos envolvemos quando estudamos álgebra abstrata? A seguir, descrevemos o que consideramos serem as principais ações envolvidas:

- Perceber, descrever, definir e representar regularidades e padrões pode ser feito por meio de palavras, símbolos, tabelas ou gráficos. Hershkowitz, Arcavi e Bruckheimer (2001) ilustram várias estratégias baseadas em perceber, descrever e representar padrões que apóiam a resolução desta tarefa.

- Lidar com expressões simbólicas de acordo com as regras algébricas, com um determinado propósito (por exemplo, substituir números por variáveis, simplificar, resolver), é uma ação mais comum na álgebra, especialmente no contexto de abstrair para uma situação particular para uma generalização.

- A tradução da simbologia e a leitura consistem na inspeção de expressões em linguagem algébrica para extrair informações delas.

- Conectar representações consiste em usar várias representações para implantar ideias e conceitos, para operá-las, discernir nuances e gerar novos conhecimentos sobre conteúdo matemático (diSESSA, HAMMER e SHERIN, 1991). Traduzir dentro e entre representações também pode influenciar tanto a compreensão de um conceito quanto a geração de uma solução para um problema.

- Criar, apresentar situações ou expressões para um propósito desejado é comum nos estágios iniciais da modelagem: é preciso decidir sobre uma variável a ser escolhida (ARCAVI, 1994) e, em seguida, criar expressões, equações ou desigualdades apropriadas para estabelecer relações que serão instrumentais na resolução do problema modelado.

O que foi exposto se aplica naturalmente ao estudo de simetria. A simetria tornou-se importante e fundamental em diversas áreas do 
Grupos diedrais: uma proposta concreta para uma apresentação inicial da Álgebra Abstrata para licenciandos em Matemática

co-nhecimento como Arquitetura, Artes, Biologia, Física, Química, Mate-mática, Literatura, entre outras.

No século XIX, Evariste Galois formalizou o conceito de Simetria baseado na ideia de grupos de transformações. A ideia de grupo é usada para se obter uma estrutura no conjunto das transformações isométricas de um objeto em si mesmo.

O estudo, na Álgebra Abstrata, de rotações e translações em polígonos regulares é conhecido como Grupos Diedrais. Em Matemática, a Teoria dos Grupos é um ramo do que é conhecido como Estruturas Algébricas.

Quando buscamos a solução de uma equação algébrica de grau maior ou igual a 5 , a solução nem sempre se encontra por meio dos radicais, conforme Vieira (2015),

[...] equações algébricas de grau até quatro são solúveis por radicais. Entretanto, isso não é valido em geral para uma equação algébrica de grau $n \geq 5$. Prova-se, usando o conceito de grupo (como também o conceito de extensão de corpo) que, em geral uma equação algébrica de quinto grau não é solúvel por radicais, ou seja, não há fórmulas para determinar as raízes de uma equação arbitrária de grau $n \geq 5$. (VIEIRA, 2015, p. 145).

Assim sendo, Galois procurou descrever as simetrias das equações satisfeitas pelas soluções de uma equação polinomial. De acordo com Vieira (2015), Galois associou um grupo a cada polinômio e usou propriedades desse grupo para dar condições para que a equação algébrica associada ao polinômio seja solúvel por radicais.

De acordo com Stewart (2012), uma simetria de um objeto matemático é uma transformação que preserva a estrutura do objeto. Deste modo, a simetria de uma figura geométrica é um rearranjo da figura preservando a disposição de seus lados e vértices, bem como suas distâncias e ângulos.

Um mapa do plano para preservar a simetria de um objeto é chamado de movimento rígido. A partir das observações em cada rearranjo, busca-se conhecer as relações entre o abstrato e o concreto na teoria dos grupos. Mas, para que se entenda o comportamento das fi- 
Grupos diedrais: uma proposta concreta para uma apresentação inicial da Álgebra Abstrata para licenciandos em Matemática

guras e suas simetrias, é preciso um conhecimento matemático com uma ênfase muito grande na abstração, que é o conceito de grupos.

Através do conceito de grupo, foi possivel perceber que a simetria é um importante conceito matemático e, por meio da mesma, era possível fazer cálculos, conforme Stewart (2012),

A simetria não era mais uma vaga impressão de regularidade ou uma sensação artística de elegância e beleza. Era um nítido conceito matemático com uma rigorosa definição lógica. Era possivel fazer cálculos com simetrias e demonstrar teoremas sobre ela. Nascia uma nova área: a teoria dos grupos [...]. (STEWART, 2012, p.151).

Domingues e Iezzi (2003) afirmam que a ideia de Grupo é um instrumento da mais alta importância para a organização e o estudo de muitas partes da matemática. E por fazer parte da abstração permite fazer generalizações que possibilitam outros caminhos levando-os a uma construção de ideias e conceitos mais coerentes. Ainda mais, na Álgebra Abstrata, que tem como objetivo a descoberta de verdades sobre sistemas algébricos, dos quais são formados por um conjunto com uma ou mais operações binárias independentes da especificidade das operações, têm propriedades e são nelas que as simetrias são exploradas a fim de deduzir as consequências de seus rearranjos.

No estudo sobre Teoria dos grupos, em especial, o grupo de simetrias é observado que, para todo poligono regular de $n$ lados quaisquer, representado por $D n$, existem relações/combinações que permitem ser visualizadas a partir do concreto. O que facilita o ensino-aprendizagem, pois o aluno pode manipular, manualmente e visualmente, cada relação e combinação e sua identificação as simetrias, numa figura, é da maior relevância na investigação das propriedades dela e na resolução de problemas geométricos que lhe dizem respeito (ARAÚJO, 2017).

Neste artigo, nosso interesse é salientar a importância da formalização do conceito de grupo utilizando a simetria dos grupos diedrais. É o que será exposto a seguir. 
Grupos diedrais: uma proposta concreta para uma apresentação inicial da Álgebra Abstrata para licenciandos em Matemática

\section{Simetrias do Triângulo Equilátero e do Quadrado}

\subsection{Triângulo Equilátero}

Por definição, temos um triângulo equilátero quando este possuir todos os lados de mesma medida. Consequentemente, é equiangular, isto é, possui todos os ângulos internos congruentes e medem $60^{\circ}$.

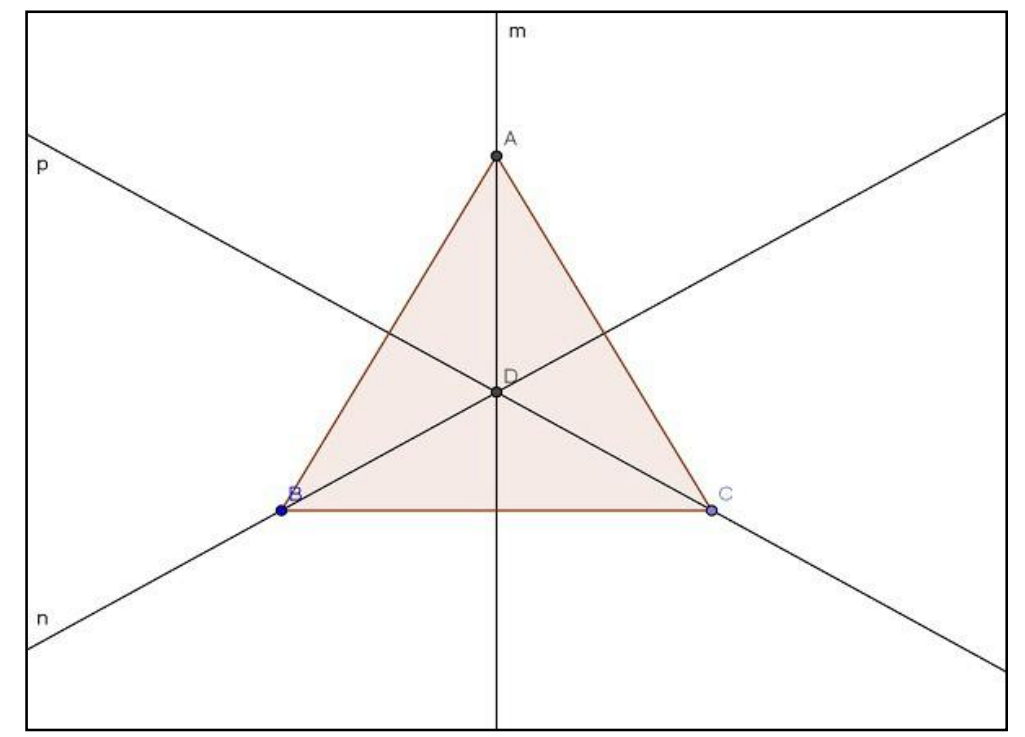

Figura 1 - Triângulo equilátero

Fonte: Autores (2019)

Suponhamos que removemos uma região triangular de um plano - movê-la, de alguma forma - então, colocamos o triângulo de volta para o espaço que ocupou originalmente. Nosso objetivo, agora, é descrever todas as maneiras possiveis em que isso pode ser feito. Mais especificamente, queremos descrever as possiveis relações entre a posição de partida do triângulo e sua posição final em termos de movimentos através dos seus três eixos de simetrias. No entanto, estamos interessados no efeito final de um movimento e não no próprio movimento. Assim, por exemplo, a partir de uma figura inicial, consideremos uma rotação de $120^{\circ}$ e uma rotação de $480^{\circ}$, elas representam o mesmo 
Grupos diedrais: uma proposta concreta para uma apresentação inicial da Álgebra Abstrata para licenciandos em Matemática

movimento, uma vez que têm o mesmo efeito final em cada ponto. Com esta convenção simplificadora, podemos alcançar nosso objetivo.

Assim, seja um triângulo equilátero $\mathrm{ABC}$, onde $m$, $n$ e $p$ são as mediatrizes relativas aos lados $\mathrm{BC}, \mathrm{AC}$ e $\mathrm{AB}$. Logo, os movimentos de translação, em torno de cada eixo de simetria axial, denotamos por: $\sigma m, \sigma n$ e $\sigma p$ serão representados a seguir.

$$
\sigma m=\left[\begin{array}{lll}
A & B & C \\
A & C & B
\end{array}\right] \sigma n=\left[\begin{array}{lll}
A & B & C \\
B & A & C
\end{array}\right] \sigma p=\left[\begin{array}{lll}
A & B & C \\
C & B & A
\end{array}\right]
$$
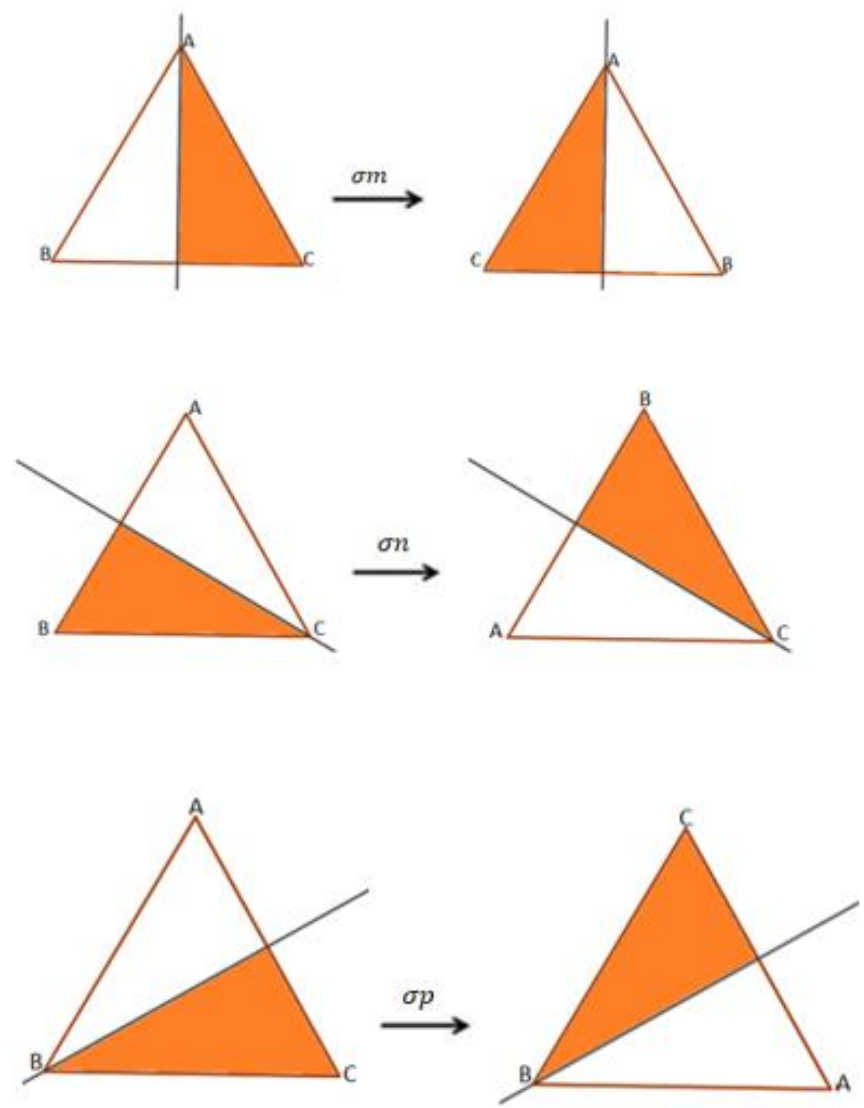

Figura 2 - Translações $\sigma m$, $\sigma n$ e $\sigma p$ Fonte: Autores (2019)

Ao rotacionarmos o triângulo equilátero, sob os ângulos de $0^{\circ}$, $120^{\circ}$ e $240^{\circ}$, em torno de $\mathrm{D}$ (centro do triângulo equilátero), no sentido anti-horário teremos: 
Grupos diedrais: uma proposta concreta para uma apresentação inicial da Álgebra Abstrata para licenciandos em Matemática

$$
I=\left[\begin{array}{lll}
A & B & C \\
A & B & C
\end{array}\right] R_{120^{\circ}}=\left[\begin{array}{lll}
A & B & C \\
C & A & B
\end{array}\right] R_{240^{\circ}}=\left[\begin{array}{lll}
A & B & C \\
B & C & A
\end{array}\right]
$$
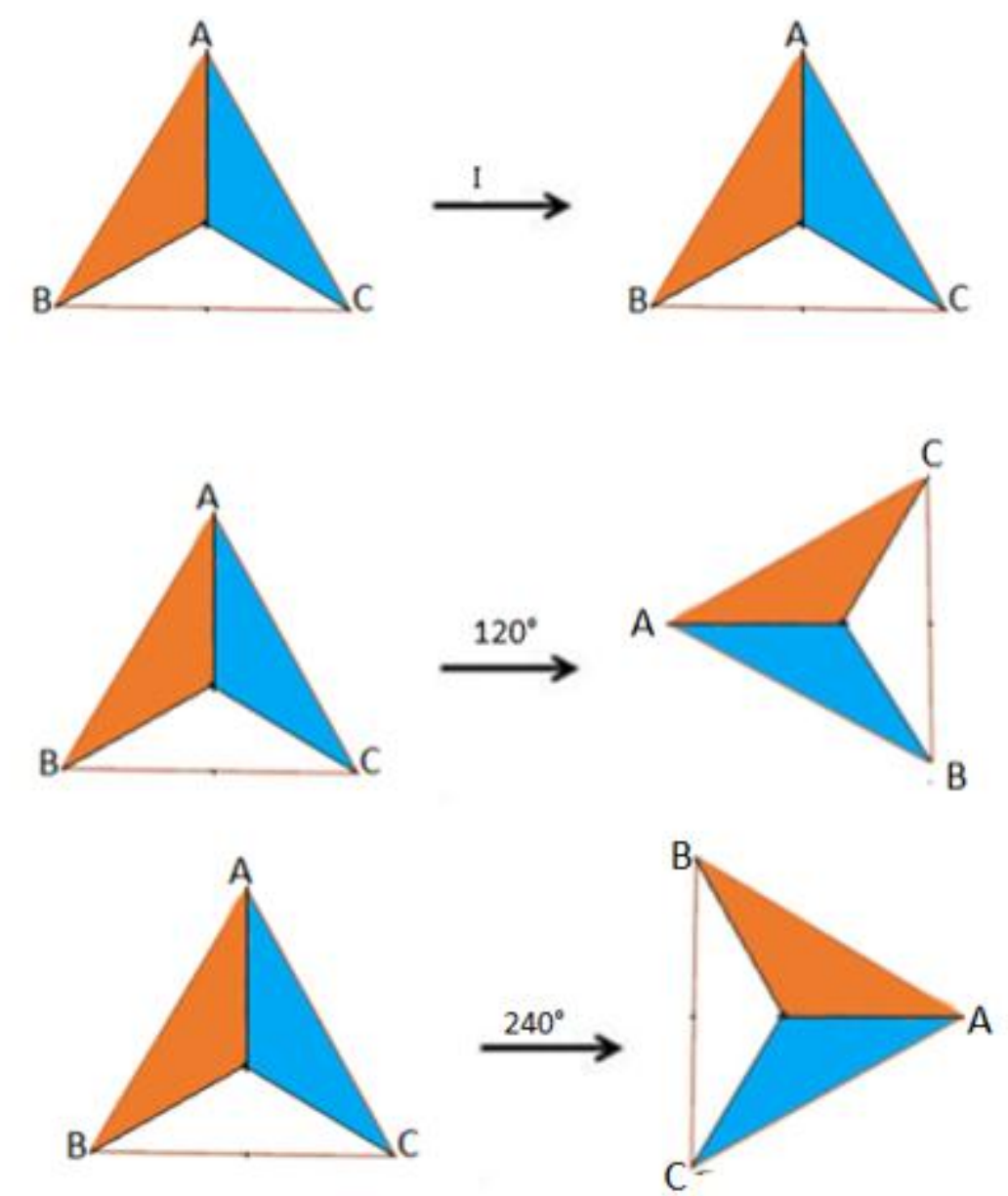

Figura 3 - Rotações $0^{\circ}, 120^{\circ}$ e $240^{\circ}$

Fonte: Autores (2019)

Observe as figuras 2 e 3 , afirmamos, agora, que qualquer movimento - não importa o quão complicado seja - é equivalente a um desses seis. Para verificar essa afirmação, observe que a posição final do triângulo é completamente determinada pela localização e orientação (isto é, virada para cima ou virada para baixo) de qualquer canto em particular. Mas, claramente, há somente três posições e três orienta- 
Grupos diedrais: uma proposta concreta para uma apresentação inicial da Álgebra Abstrata para licenciandos em Matemática

ções para um determinado canto, assim há exatamente seis posições finais distintas para o canto.

Para encontrar uma simetria do $\triangle A B C$, devemos primeiro examinar as permutações dos vértices $A, B$ e $C$ e, em seguida, perguntar se uma permutação se estende a uma simetria do triângulo. Lembre-se de que a permutação de um conjunto $S$ é um para um e no mapa $\pi$ : $S$ $\rightarrow S$. Os três vértices têm $3 !=6$ permutações; então, o triângulo tem, no máximo, seis simetrias. Para verificar se existem seis permutações, observe que existem três possibilidades diferentes para o primeiro vértice e duas para o segundo, e o vértice restante é determinado pela colocação dos dois primeiros. Portanto, temos 3.2.1=3! = 6 arranjos diferentes. Para denotar a permutação dos vértices de um triângulo equilátero que envia $A$ para $B, B$ para $C$ e $C$ para $A$, escrevemos a matriz

$$
\left[\begin{array}{lll}
A & B & C \\
C & A & B
\end{array}\right]
$$

Observe que essa permutação específica corresponde ao movimento rígido de rotação $R_{120}$ no sentido anti-horário. De fato, toda permutação dá origem a uma simetria do triângulo. Uma pergunta natural a se fazer é: o que acontece se um movimento do triângulo $\triangle A B C$ é seguido por outro? Qual simetria é $\mathrm{R}_{120} \sigma n$ ? Isto é, o que acontece quando fazemos a permutação $\mathrm{R}_{120}$ e depois a permutação on? Lembre-se de que estamos compondo funções neste caso. Embora, geralmente, façamos a multiplicação da esquerda para a direita, compomos funções da direita para a esquerda.

Vamos observar, através da tabela de operação, as composições dessas transformações no plano: 
Grupos diedrais: uma proposta concreta para uma apresentação inicial da Álgebra Abstrata para licenciandos em Matemática

Tabela 1 - Tabela de Composição do triângulo

\begin{tabular}{|c|c|c|c|c|c|c|}
\hline 0 & I & $R_{120^{\circ}}$ & $R_{240^{\circ}}$ & $\sigma m$ & on & $\sigma p$ \\
\hline I & I & $R_{120^{\circ}}$ & $R_{240^{\circ}}$ & $\sigma m$ & $\sigma n$ & $\sigma p$ \\
\hline$R_{120^{\prime}}$ & $R_{120^{\circ}}$ & $R_{240^{\circ}}$ & I & $\sigma p$ & $\sigma m$ & $\sigma n$ \\
\hline$R_{240^{\circ}}$ & $R_{240^{\circ}}$ & I & $R_{120^{\circ}}$ & $\sigma n$ & $\sigma p$ & $\sigma m$ \\
\hline$\sigma m$ & $\sigma m$ & $\sigma n$ & $\sigma p$ & I & $R_{120^{2}}$ & $R_{240^{\circ}}$ \\
\hline$\sigma n$ & $\sigma n$ & $\sigma p$ & $\sigma m$ & $R_{240^{\circ}}$ & I & $R_{120^{2}}$ \\
\hline$\sigma p$ & $\sigma p$ & $\sigma m$ & $\sigma n$ & $R_{120^{\circ}}$ & $R_{240^{\circ}}$ & I \\
\hline
\end{tabular}

Fonte: Autores (2019)

O grupo $D=\left\{I_{1} R_{120^{3}}, R_{240^{s}}, \sigma m, \sigma n, \sigma p\right\}$ é o grupo de simetrias do triângulo equilátero.

De maneira semelhante, vamos analisar as simetrias do quadrado.

\subsection{Quadrado}

Por definição temos que, um quadrado (figura 4) é um retângulo que possui todos os lados de mesma medida. 
Grupos diedrais: uma proposta concreta para uma apresentação inicial da Álgebra Abstrata para licenciandos em Matemática

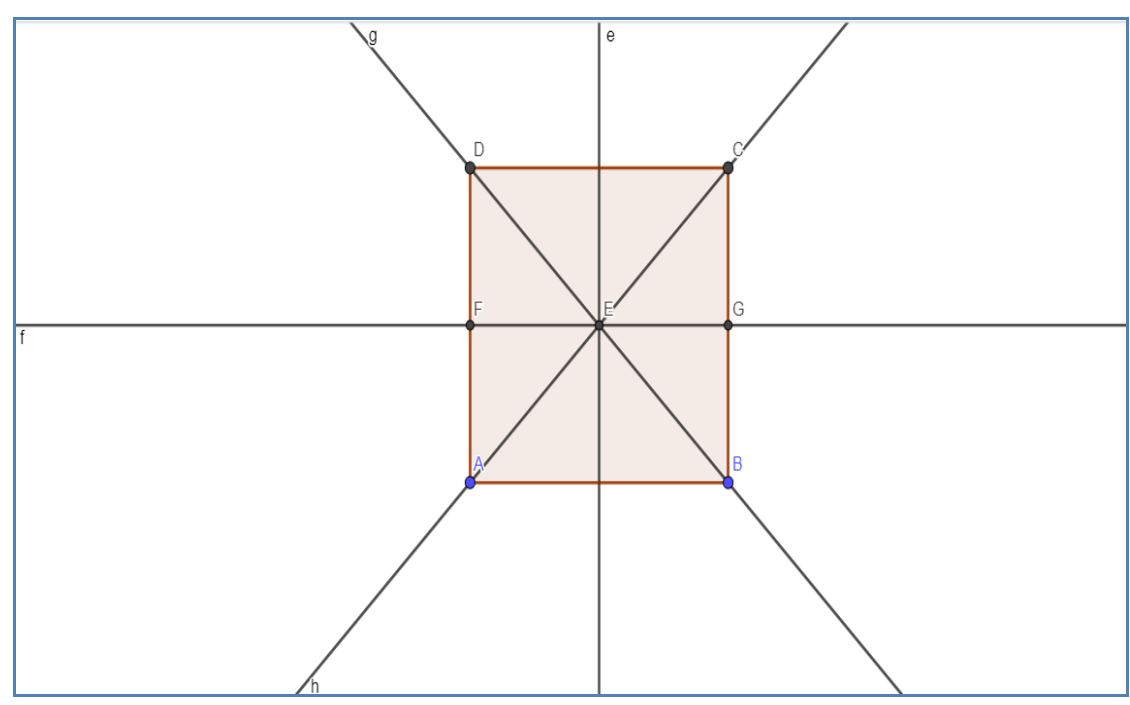

Figura 4 - Quadrado

Fonte: Autores (2019)

Assim, seja um quadrado $\mathrm{ABCD}$, onde $f$ e $e$ são as mediatrizes dos lados $\mathrm{AD}$ e DC, respectivamente, $g$ e $h$ são as diagonais do quadrado e consideramos uma rotação de $90^{\circ}$ e uma rotação de $450^{\circ}$ iguais, uma vez que têm o mesmo efeito líquido em cada ponto.

Observe as figuras 5 e 6, afirmamos, agora, que qualquer movimento - não importa o quão complicado seja - é equivalente a um desses oitos. Para verificar essa afirmação, observe que a posição final do quadrado é completamente determinada pela localização e orientação (isto é, virada para cima ou virada para baixo) de qualquer canto em particular. Mas, obviamente, há somente quatro posições e duas orientações para um determinado canto, assim, há exatamente oito posições finais distintas para o canto - temos que, os movimentos de translação em torno dos eixos de simetria axial serão representados a seguir:

$$
\begin{aligned}
& r_{e}=\left[\begin{array}{llll}
A & B & C & D \\
B & A & D & C
\end{array}\right] \quad r_{f}=\left[\begin{array}{llll}
A & B & C & D \\
D & C & B & A
\end{array}\right] \\
& r_{g}=\left[\begin{array}{llll}
A & B & C & D \\
C & B & A & D
\end{array}\right] \quad r_{h}=\left[\begin{array}{llll}
A & B & C & D \\
A & D & C & B
\end{array}\right]
\end{aligned}
$$


Grupos diedrais: uma proposta concreta para uma apresentação inicial da Álgebra Abstrata para licenciandos em Matemática
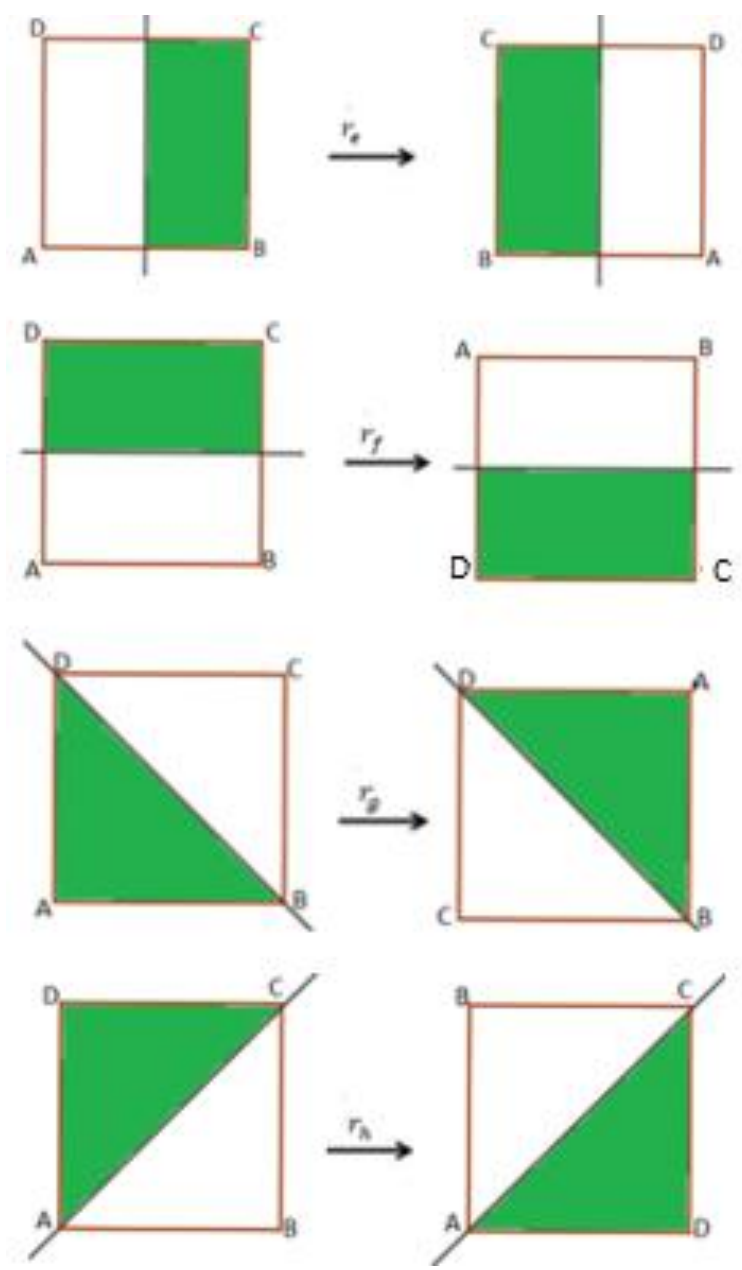

Figura 5 - Translações do quadrado Fonte: Autores (2019)

$r_{f}=$ Virar em torno de um eixo horizontal

$r_{e}=$ Virar em torno de um eixo vertical

$r_{g}=$ Virar em torno de uma diagonal

$r_{h}=$ Virar em torno de outra diagonal

Além disso, se rotacionarmos o quadrado sob os ângulos de $0^{\circ}$, $90^{\circ}, 180^{\circ}$ e $270^{\circ}$, em torno de $\mathrm{E}$ (centro do quadrado), no sentindo anti-horário, teremos: 
Grupos diedrais: uma proposta concreta para uma apresentação inicial da Álgebra Abstrata para licenciandos em Matemática

$$
I=\left[\begin{array}{llll}
A & B & C & D \\
A & B & C & D
\end{array}\right] \quad R_{90^{\circ}}=\left[\begin{array}{llll}
A & B & C & D \\
D & A & B & C
\end{array}\right]
$$

$$
R_{180^{\circ}}=\left[\begin{array}{llll}
A & B & C & D \\
C & D & A & B
\end{array}\right] \quad R_{270^{\circ}}=\left[\begin{array}{llll}
A & B & C & D \\
B & C & D & A
\end{array}\right]
$$
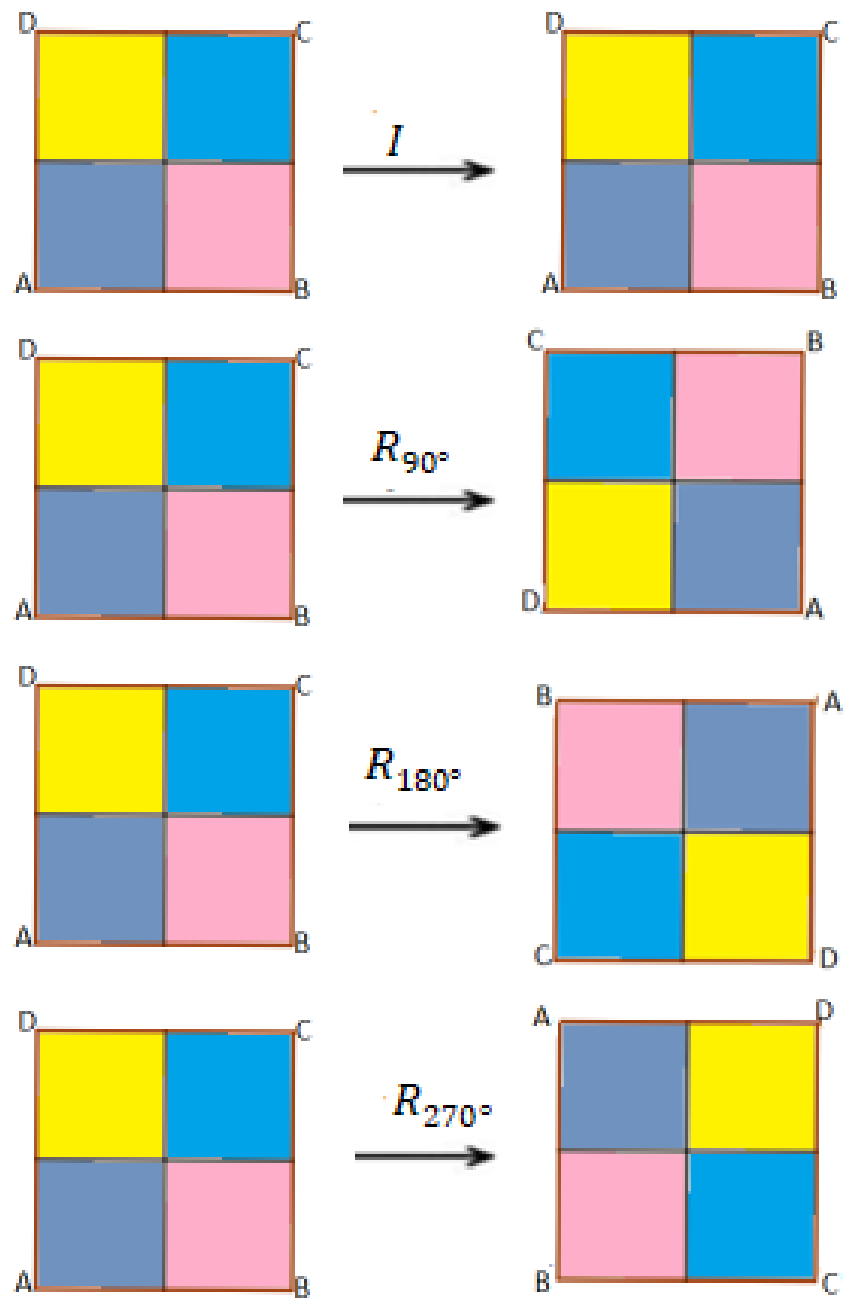

Figura 6 - Rotações no quadrado

Fonte: Autores (2019)

Vamos investigar algumas consequências do fato de que cada movimento é igual a um dos oito listrados nas figuras anteriores. Suponhamos que um quadrado é reposicionado por uma rotação de $90^{\circ}$, 
Grupos diedrais: uma proposta concreta para uma apresentação inicial da Álgebra Abstrata para licenciandos em Matemática

seguida por uma virada em torno do eixo horizontal de simetria, conforme a figura 7 .
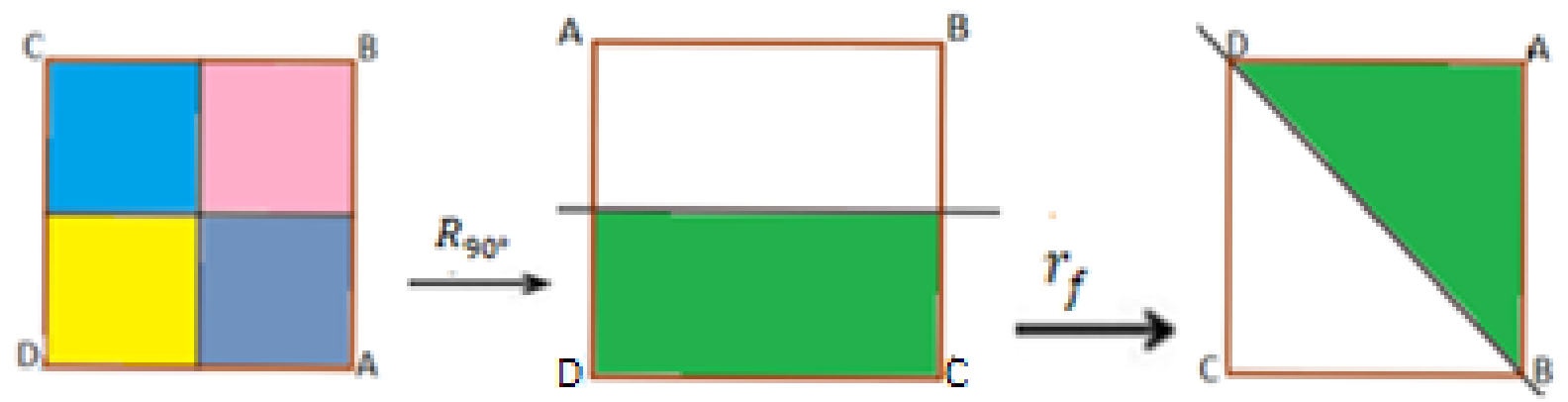

Figura 7 - Rotação de $90^{\circ}$ com uma translação na horizontal

Fonte: Autores (2019)

Assim, vemos que este par de movimentos tomados em conjunto é igual a um único movimento $r_{g}$. Esta observação sugere que é possível compor dois movimentos para obter um único movimento $\mathrm{E}$, na verdade, podemos fazê-los, uma vez que os oito movimentos podem ser vistos como funções da região quadrada para si mesma e, como tal, podemos combiná-los usando a composição da função.

Os oito movimentos $R_{0}, R_{90}, R_{180}, R_{270}, r_{e}, r_{f}, r_{g}$ e $r_{h}$, juntamente com a operação de composição, formam um sistema matemático denominado grupo diedral de ordem 8 (a ordem de um grupo é o número de elementos que ele contém).

Para facilitar os cálculos, construímos uma tabela de operação para $\mathrm{D}_{4}$, a seguir (Tabela 2). A entrada circundada representa o fato de que $r_{g}=\mathrm{R}_{90} r_{f}$. (Em geral $a b$, denota a entrada na interseção da coluna $a$, à esquerda, e a linha $b$, na parte superior). 
Grupos diedrais: uma proposta concreta para uma apresentação inicial da Álgebra Abstrata para licenciandos em Matemática

Tabela 2 - Tabela de Cayley do quadrado

\begin{tabular}{|c|c|c|c|c|c|c|c|c|}
\hline . & I & $R_{90^{3}}$ & $R_{180^{3}}$ & $R_{270^{\circ}}$ & $r_{B}$ & $r_{f}$ & $r_{g}$ & $r_{h}$ \\
\hline I & I & $R_{90^{3}}$ & $R_{180^{3}}$ & $R_{270^{\circ}}$ & $r_{e}$ & $r_{f}$ & $r_{g}$ & $r_{h}$ \\
\hline$R_{90^{3}}$ & $R_{90^{\circ}}$ & $R_{180^{\circ}}$ & $R_{270^{\circ}}$ & I & $r_{h}$ & $r_{g}$ & $r_{B}$ & $r_{f}$ \\
\hline$R_{180^{\circ}}$ & $R_{180^{\circ}}$ & $R_{270^{\circ}}$ & I & $R_{90^{\circ}}$ & $r_{f}$ & $r_{\theta}$ & $r_{h}$ & $r_{g}$ \\
\hline$R_{270^{\circ}}$ & $R_{270^{\circ}}$ & I & $R_{90^{\circ}}$ & $R_{180^{\circ}}$ & $r_{g}$ & $r_{h}$ & $r_{f}$ & $r_{e}$ \\
\hline$r_{\theta}$ & $r_{B}$ & $r_{g}$ & $r_{f}$ & $r_{h}$ & $I$ & $R_{180^{\circ}}$ & $R_{90^{\circ}}$ & $R_{270^{\circ}}$ \\
\hline$r_{f}$ & $r_{f}$ & $r_{h}$ & $r_{e}$ & $r_{g}$ & $R_{180^{\circ}}$ & I & $R_{270^{\circ}}$ & $R_{90^{\circ}}$ \\
\hline$r_{g}$ & $r_{g}$ & $r_{f}$ & $r_{h}$ & $r_{B}$ & $R_{270^{\circ}}$ & $R_{90^{\circ}}$ & I & $R_{180^{\circ}}$ \\
\hline$r_{h}$ & $r_{h}$ & $r_{e}$ & $r_{g}$ & $r_{f}$ & $R_{90^{3}}$ & $R_{270^{\circ}}$ & $R_{180^{\circ}}$ & I \\
\hline
\end{tabular}

Fonte: Autores (2019)

Observe quão ordenada esta tabela parece! Isso não é um acidente. Talvez, a característica mais importante desta tabela seja que ela foi completamente preenchida sem introduzir novos movimentos. Natural-mente, isto é, porque, como já assinalamos, qualquer sequência de movimentos acaba por ser a mesma de um destes oito. 
Grupos diedrais: uma proposta concreta para uma apresentação inicial da Álgebra Abstrata para licenciandos em Matemática

\section{Grupos Diedrais}

As análises realizadas anteriormente, para um triângulo equilátero e para o quadrado, também podem ser feitas para um pentágono regular ou, de fato, qualquer $n$-gon regular $(n \geq 3)$. O grupo correspondente é indicado por Dn e é chamado de Grupo Diedral. O conjunto Dn das simetrias de um polígono regular de $\mathrm{n}$ lados é um grupo com a operação composição de aplicações. O grupo (Dn, ${ }^{\circ}$ ) de ordem $2 \mathrm{n}$ e, constituído de $\mathrm{n}$ rotações e $\mathrm{n}$ translações.

Os grupos diedrais surgem, frequentemente, na arte e na natureza. Muitos dos desenhos decorativos, usados em revestimentos de pisos, cerâmica e edifícios têm um dos grupos diedrais como um grupo de sime-tria.

De acordo com Cançado (2016), o Grupo Diedral ou Grupo de Simetrias pode ser entendido como Grupo de Permutações. Isto porque, existe uma bijeção $f$ de $D n$ em $D n$ e, os movimentos de rotação e reflexão de Dn são representados por permutações.

Algebricamente, isto diz que se $A$ e $B$ estão em $D_{3}$, então: $A R_{0}=R_{0} A=A$. Assim, a combinação de qualquer elemento $A$ de cada lado com $R_{0}$ origina $A$, de volta. Um elemento $R_{0}$ com essa propriedade é chamada de uma identidade, e cada grupo deve ter uma. Além disso, vamos que para cada elemento $A$ em $D_{3}$, há exatamente um elemento $B$ em $D_{3}$ tal que $A B=B A=R_{0}$. Neste caso, $B$ é dito ser o inverso de $A$ e vice-versa. Por exemplo, no triângulo eqüilátero, $R_{120}$ e $R_{240}$ são inversos um do outro, e on é o seu inverso. O termo inverso é descritivo, pois se $A$ e $B$ são inversos um do outro, então $B$ "desfaz" enquanto $A$ "refaz", no sentindo de que $A$ e $B$ tomados juntos em qualquer ordem produzem $R_{O}$ indicando nenhuma alteração. Outra característica marcante da tabela é que cada elemento de $D_{3}$ e $D_{4}$ aparece exatamente uma vez em cada linha e coluna. Essa propriedade é algo que todos os grupos devem ter, e, na verdade, é bastante útil manter esse fato em mente quando se constrói a tabela pela primeira.

Outra propriedade em $D_{4}$ merece um comentário especial. Observe que $\sigma$ nom $\neq \sigma m \sigma n$, mas $R_{120} R_{240}=R_{240} R_{120}$. Assim, num grupo, $a b$ pode ou não ser o mesmo que $b a$. Se acontecer de $a b=b a$, para todas 
Grupos diedrais: uma proposta concreta para uma apresentação inicial da Álgebra Abstrata para licenciandos em Matemática

as escolhas dos elementos $a$ e $b$ do grupo, dizemos que o grupo é comutativo ou abeliano. Caso contrário, dizemos que o grupo é não abeliano.

Até agora, temos ilustrado, através de $D_{3}$, três das quatro condições que definem um grupo nomeadamente, fechamento, existência de uma identidade e existência de inversos. A condição restante requerida para um grupo é a associatividade; isto é, $(a b) c=a(b c)$ para todos $a, b, c$ no conjunto. Para ter certeza de que $D_{3}$ é realmente um grupo, devemos verificar esta equação para cada um dos $6^{3}=216$ possiveis escolhas de $a, b$ e $c$ em $D_{3}$. Na prática, no entanto, isso raramente é feito! Aqui, por exemplo, observamos simplesmente que os seis movimentos são funções e a operação é composição de função. Então, como a composição da função é associativa, não precisamos verificar as equações.

\section{Definição de Grupo}

De acordo com Vieira (2015), um grupo é uma estrutura algébrica relativamente simples, pois é uma estrutura dotada de apenas uma operação binária. Os números inteiros, módulo n, e as simetrias de um triângulo ou quadrado são exemplos de grupos. Uma operação binária ou lei de composição em um conjunto $G$ é uma função $G \times G \rightarrow G$ que atribui à cada par $(a, b) \in G \times G$ um elemento único $a \circ b$, ou $a b \mathrm{em} G$, chamado composição de $a$ e $b$. Um grupo $\left(\mathrm{G},{ }^{\circ}\right)$ é um conjunto $\mathrm{G}$, juntamente com uma lei da composição $(a, b) \rightarrow a^{\circ} b$, que satisfaz os seguintes axiomas:

- A lei da composição é associativa. Isso é, $\left(a^{\circ} b\right){ }^{\circ} c=a^{\circ}(b \circ c)$

Com $a, b, c \in G$

- Existe um elemento e $\in G$, chamado elemento de identidade, como que para qualquer elemento $a \in G$. $e^{\circ} a=a^{\circ} e=a$ 
Grupos diedrais: uma proposta concreta para uma apresentação inicial da Álgebra Abstrata para licenciandos em Matemática

- Para cada elemento $a \in G$, existe um elemento inverso em $G$, denotado por -1 , de modo que $a^{\circ} a^{-1}=a^{-1} \circ a=e$

Um grupo G com a propriedade que $a^{\circ} b=b^{\circ} a$ para todos $a, b \in$ $G$ é chamado abeliano ou comutativo. Os grupos que não satisfazem essa propriedade são considerados não-abelianos ou não-comutativos.

\section{Aplicações}

Muitas vezes, a utilidade de algum conhecimento particular em Matemática não é óbvia, nem simples. Assim é com a teoria dos grupos. Suas aplicações no mundo real surgem através de conexões que a princípio não são facilmente perceptiveis. Como, por exemplo, relacionar a teoria de grupos em conexão com a simetria molecar e as propriedades molecares estudadas na química quântica. No entanto, vamos indicar aqui alguns de seus usos nas ciências e nas artes.

A maioria das aplicações científicas de grupos está no campo da Física e da Química. Uma das aplicações mais impressionantes ocorreu em 1962. Naquele ano, os físicos Murray Gell-Mann e Yuval Ne'eman usaram a teoria de grupo para prever a existência de uma nova partícula, que foi designada a omega minus particle (A Dictionary of Physics, 2014). Não foi até 1964, que a existência desta partícula foi confirmada em experimentos de laboratório.

Um dos usos de grupos mais extensos tem sido na ciência da cristalografia. Cada figura geométrica em duas ou três dimensões têm seus movimentos rígidos associados, ou simetrias. Esta associação fornece uma conexão natural entre grupos, onde ocorrem permutações, como os diedrais, vistos neste artigo, e muitos objetos no mundo real. Uma das conexões mais frutíferas foi feita no estudo da estrutura dos cristais. Os cristais são classificados de acordo com a simetria geométrica com base em uma estrutura com um arranjo equilibrado de suas faces. Um dos exemplos mais simples e mais comuns de tal 
Grupos diedrais: uma proposta concreta para uma apresentação inicial da Álgebra Abstrata para licenciandos em Matemática

estrutura é fornecido pelo fato de que um sal de mesa comum ( $\mathrm{NaCl}$ ) cristal está na forma de um cubo (figura 8).

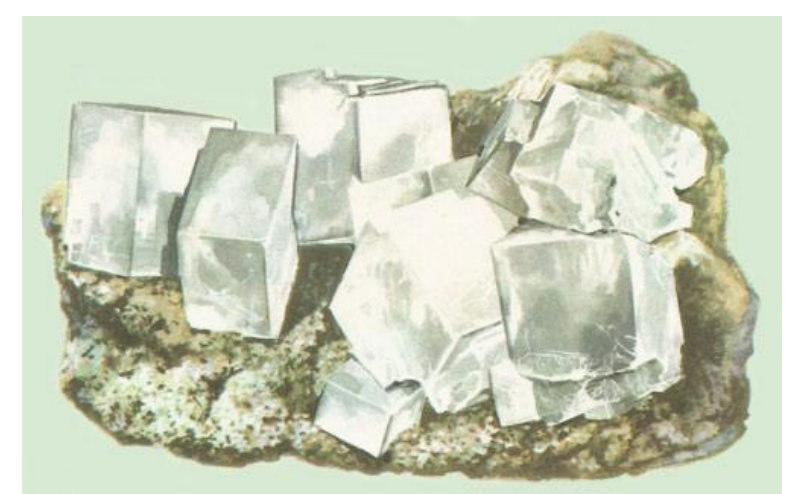

Figura 8: $(\mathrm{NaCl})$ cristal

Fonte: Oxford University Press (2014)

Neste artigo, examinamos os Grupos Diedrais que estão relacionados aos movimentos rígidos de uma figura plana. Vimos dois exemplos deste tipo de grupo. O primeiro foi o grupo diedral $D_{3}$, o grupo das simetrias de um triângulo equilátero e, o outro grupo diedral, $D_{4}$, o grupo de simetrias de um quadrado.

Não é difícil ver que as simetrias de qualquer figura plana $F$ formam um grupo para a composição de aplicações. Antes de considerarmos algumas outras figuras planas específicas $F$, vamos comentar novamente sobre o termo simetria.

De acordo com a terminologia convencional na Álgebra, usamos a palavra simetria para nos referirmos a um movimento rígido de uma figura geométrica. No entanto, o termo é comumente empregado de outra forma. Por exemplo, o pentágono mostrado na Figura 9, é dito ter simetria no que diz respeito à linha vertical $\ell$ e, através do centro $O$ e o vértice no topo, ou é simétrico em relação a $\ell$. Para fazer uma distinção entre os dois usos, utilizaremos o termo simetria geométrica para este último tipo de simetria. 
Grupos diedrais: uma proposta concreta para uma apresentação inicial da Álgebra Abstrata para licenciandos em Matemática

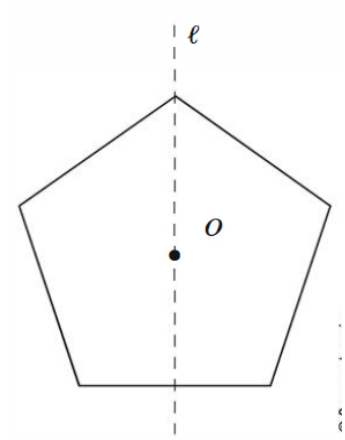

Figura 9: Pentágono

Fonte: Oxford University Press (2014)

Os grupos de simetrias para polígonos regulares com três ou quatro lados generalizam-se para um poligono regular $P$ com $n$ lados, para qualquer inteiro positivo $\mathrm{n}>4$. Qualquer simetria $f$ de $\mathrm{P}$ é determinada pelas imagens dos vértices de $P$. Sejam os vértices numerados $1,2, \ldots, n$, e consideremos a aplicação que faz com que a simetria $f$ de $\mathrm{P}$ corresponda à permutação em $\{1,2, \ldots, n\}$ que tem a forma de matriz

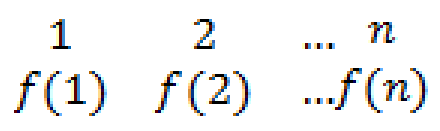

Uma vez que $f$ é completamente determinado pelas imagens dos vértices, esta aplicação é claramente uma bijetiva entre os movimentos rígidos de $P$ e $D_{n}$ é um subconjunto do grupo simétrico $S_{n}$ de todas as permutações em $\{1,2, \ldots \ldots n\}$.

Polígonos regulares com $n=5$ (um pentágono) e $n=6$ (um hexágono) são mostrados na Figura 10. Tendo em mente que uma simetria é determinada pelas imagens dos vértices, pode-se ver que $D_{n}$ consiste em $n$ rotações anti-horário e $n$ reflexões sobre uma linha através do centro $O$ de $P$.

Se $n$ é ímpar, cada reflexão é sobre uma linha através de um vértice até ponto médio do lado oposto. Se $n$ é par, metade das reflexões são sobre linhas através de pares de vértices opostos, e a 
Grupos diedrais: uma proposta concreta para uma apresentação inicial da Álgebra Abstrata para licenciandos em Matemática

outra metade são sobre linhas através de pontos médios de lados opostos. Assim, $D_{n}$ tem ordem $2 n$, ou seja, $2 n$ elementos.
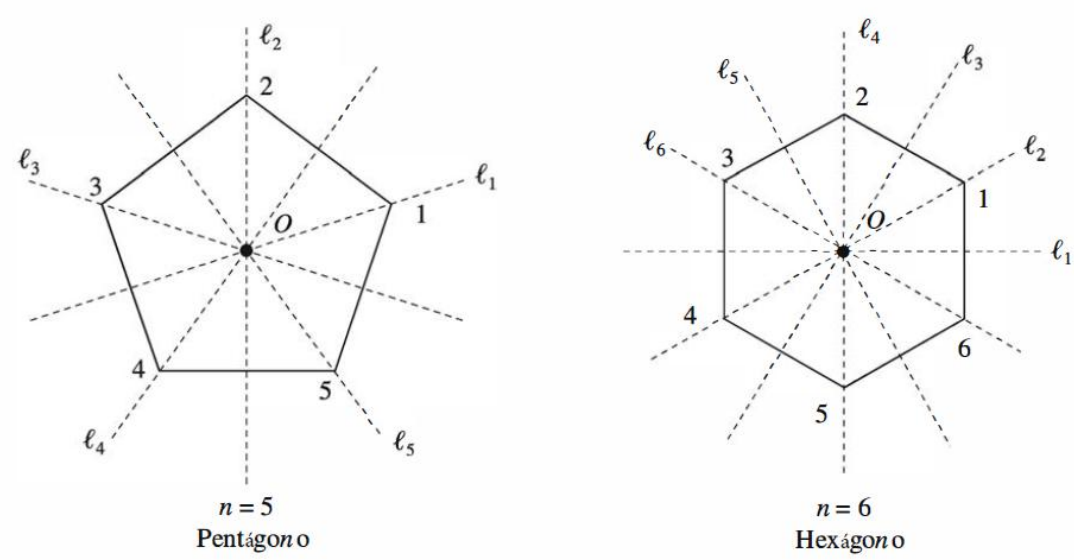

Figura 10: pentágono e hexágono Fonte: Oxford University Press (2014)

Todas as simetrias apresentadas neste artigo foram rotações ou reflexões sobre uma linha. Isso não é por acaso, porque estes são os únicos tipos de simetrias que existem para um conjunto não vazio delimitado.

Se o grupo de simetrias de uma determinada figura contém uma rotação diferente da aplicação identidade, então a figura é dita possuir simetria rotacional. Uma figura com um grupo de simetrias que inclui uma reflexão sobre uma linha é dita ter simetria reflexiva. Vejamos um exemplo.

$\mathrm{Na}$ ilustração 11 , cada parte de qualquer uma das figuras apresentadas tem um grupo de simetrias que consiste inteiramente em rotações e, cada uma, possui apenas simetria rotacional. Em contraste, o grupo de simetrias do pentágono contém reflexões e rotações, e o pentágono tem simetria reflexiva e simetria rotativa. 
Grupos diedrais: uma proposta concreta para uma apresentação inicial da Álgebra Abstrata para licenciandos em Matemática

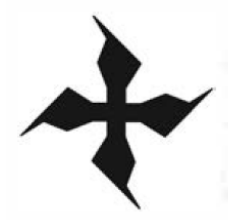

(a)

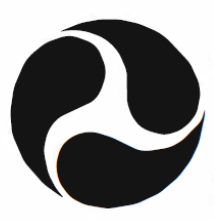

(b)

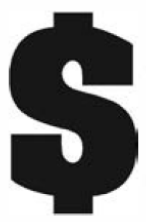

(c)

Figura 11 - Grupo de simetrias

Fonte: Oxford University Press (2014)

Como exemplo final nesta seção, consideramos o grupo de simetrias de um conjunto ilimitado. O conjunto é mostrado na Figura 12 e é composto por uma sequência horizontal de cópias da letra $R$, igualmente espaçada em 1 unidade, desde o início de um $R$ para o início do próximo $R$, e sem fim em ambas as direções.

$$
\cdots \stackrel{\mathbf{R}}{\stackrel{1 \text { unidade }}{\longrightarrow}} \mathbf{R} \quad \mathbf{R} \quad \mathbf{R} \quad \mathbf{R} \quad \mathbf{R} \cdots
$$

Figura 12 - Sequência da letra R

Fonte: Oxford University Press (2014)

Esses tipos de grupos de simetrias são comumente usados para papel de parede, padrões têxteis, cerâmicas, fitas e todos os tipos de arte decorativa. A conexão excepcional entre grupos e arte é fornecida pelas famosas obras do grande artista holandês Maurits Cornelis Escher (1898-1972).

\section{Considerações finais}

Seguindo o que foi exposto, este artigo foi uma tentativa inicial de abordar uma problemática: a centralidade da Álgebra Abstrata nos currículos dos cursos de Licenciatura em Matemática nas universidades em contraste com as muitas dificuldades que a disciplina apresenta para os licenciandos e a subsequente relutância em estudála. 
Grupos diedrais: uma proposta concreta para uma apresentação inicial da Álgebra Abstrata para licenciandos em Matemática

Foram sugeridas algumas atividades, que consideramos interessantes, dentro do conteúdo apresentado, que estão disponíveis no anexo.

Para uma discussão mais aprofundada, que não cabe no escopo deste artigo, duas questões merecem ser pesquisadas: qual é a essência da Álgebra Abstrata ensinada nos cursos de Licenciatura em Matemática e por que é ensinada? Sem a pretensão de dar aqui as repostas, ao propormos uma primeira apresentação da Álgebra Abstrata através das rotações e translações do triângulo equilátero e do quadrado, para introduzir o conceito de grupo diedral e, a partir desse grupo, generalizar propriedades válidas para qualquer grupo, propusemos possiveis respostas para as questões colocadas, fornecendo exemplos para apoiar e ilustrar os argumentos.

Através dos grupos diedrais, revisitamos e exemplificamos a natureza do assunto (objetivos, ações e entidades da álgebra abstrata) bem como o seu valor educativo e as razões plausiveis para estudá-la. Reiteramos que, este artigo foi apenas um tipo elementar de um primeiro passo para a tentativa de resolver a problemática, para o benefício dos licenciandos, professores e responsáveis pelo currículo. O artigo também incluiu tarefas e atividades interessantes para exploração e discussão para descompactar e esclarecer as questões.

Procuramos colocar alguns dos principais pontos que, em nossa prática, procuramos transmitir aos licenciandos, através de conversas explícitas ou através do trabalho em sala de aula, a partir de exemplos e problemas.

Finalizando, para uma resposta simples à incômoda e persistente pergunta "Por que eu deveria aprender álgebra abstrata?" - é porque:

álgebra é o início de uma jornada que the dá as habilidades para resolver ... problemas complexos ... Álgebra é um trampolim para aprender sobre o universo maravilhoso em que vivemos. Com ela você tem as ferramentas para entender muitas coisas e você também tem as habilidades necessárias para continuar e aprender e entender novos conteúdos matemáticos que são essenciais para explorar outros tipos de problemas e fenômenos ao nosso redor. (GIBSON, n.d.). 
Grupos diedrais: uma proposta concreta para uma apresentação inicial da Álgebra Abstrata para licenciandos em Matemática

\section{Referências}

A DICTIONARY of Physics. Oxford: Oxford University Press, 2014.

ARCAVI, Abrahan. Symbol sense: Informal sense-making in formal mathematics. For the learning of Mathematics, 14(3), p. 24-35, 1994.

CANÇADO, Ana Paula. Grupo Diedral: o estudo de grupos de simetrias em poligonos regulares. Monografia apresentada na Universidade Federal de São João del-Rei, 2016.

DAMBRÓS, Andressa. Um estudo sobre simetrias e grupos de Galois: utilizando uma via estética para acesso ao conhecimento matemático. Monografia apresentada no Centro de Ciências Tecnológicas, Universidade do Estado de Santa Catarina, 2013.

DISESSA, Andrea; HAMMER, David; SHERIN, Bruce. Inventing graphing: Meta-representational expertise in children. Journal of Mathematical Behavior, 10, p. 117-160, 1991.

DOMINGUES, Hygino H.; IEZZI, Gelson. Álgebra Moderna. 4. ed. São Paulo: Atual, 2003.

FONSECA, Rubens Vilhena. Notas de Álgebra II. Pará: Universidade do Estado do Pará, 2019. (no prelo).

FRANCO, Marcia Cristina Lemos Guimarães. Simetria. 2015. 123 f. Dissertação (Mestrado Profissional em Ensino de Matemática) - Universidade Estadual de Campinas, Campinas, 2015.

GIBSON, Jason. Why Learn Algebra? (n. d.). Math Goodies. Disponivel em:

www.mathgoodies.com/articles/why_learn_algebra.html. Acesso em: 12 nov. 2019. 
Grupos diedrais: uma proposta concreta para uma apresentação inicial da Álgebra Abstrata para licenciandos em Matemática

GRANDO, Claudia Maria. O Concreto, o abstrato e o formal no discurso e na ação pedagógica dos acadêmicos de prática de ensino em matemática da UNOESC - Chapecó. 2000. 101 f. Dissertação (Mestrado em Educação) - Universidade Federal de Santa Catarina, Florianópolis, 2000.

HERSHKOWITZ, Rina; ARCAVI, Abrahan, \& BRUCKHEIMER, Maxim. Reflections on the status and nature of visual reasoning - the case of the matches. International Journal of Mathematical Education in Science and Technology, 32(2), p. 255-265. 2001.

Online Etymology Dictionary (2001-2015).

Disponível em: www.etymonline.com. Acesso: 12 nov. 2018.

STEWART, Ian. Uma história da simetria na matemática. Tradução Claudio Carina. Rio de Janeiro: Zahar, 2012.

VIEIRA, Vandenberg Lopes. Álgebra Abstrata para Licenciatura. 2. ed. Campina Grande: EDUEPB, 2015. 
Grupos diedrais: uma proposta concreta para uma apresentação inicial da Álgebra Abstrata para licenciandos em Matemática

\section{ANEXO}

\section{Sugestões de Atividades interessantes}

1. Descreva em figuras ou palavras os elementos de $\mathrm{D}_{5}$ (simetrias do 5-gon regular ou pentágono regular).

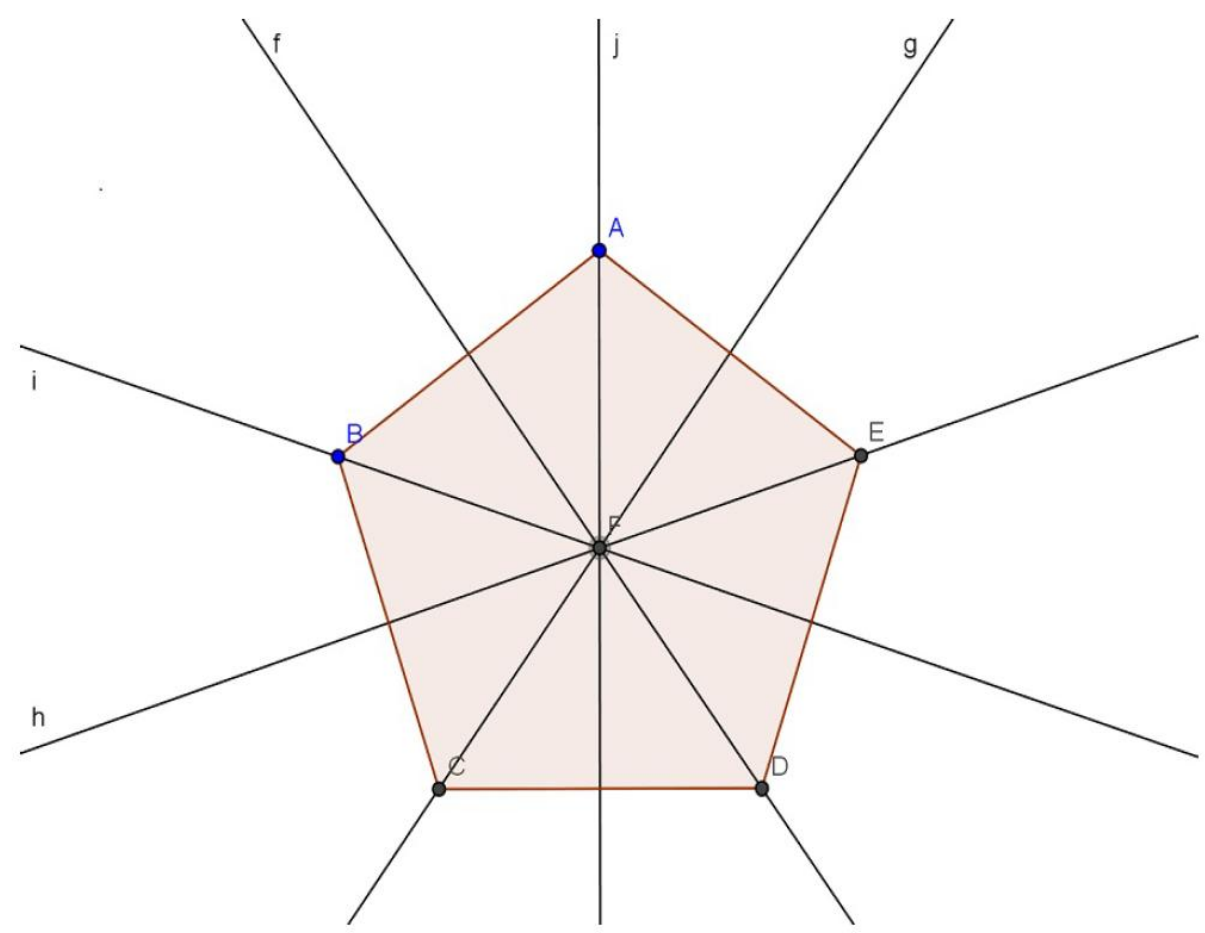


Grupos diedrais: uma proposta concreta para uma apresentação inicial da Álgebra Abstrata para licenciandos em Matemática

\begin{tabular}{|c|c|c|c|c|c|c|c|c|c|c|}
\hline$\circ$ & $I$ & $R_{72^{\circ}}$ & $R_{144^{\circ}}$ & $R_{216^{\circ}}$ & $R_{288^{\circ}}$ & $r_{j}$ & $r_{g}$ & $r_{h}$ & $r_{i}$ & $r_{f}$ \\
\hline$I$ & $I$ & $R_{72^{\circ}}$ & $R_{144^{\circ}}$ & $R_{216^{\circ}}$ & $R_{288^{\circ}}$ & $r_{j}$ & $r_{g}$ & $r_{h}$ & $r_{i}$ & $r_{f}$ \\
\hline$R_{72^{\circ}}$ & $R_{72^{\circ}}$ & $R_{144^{\circ}}$ & $R_{216^{\circ}}$ & $R_{288^{\circ}}$ & $I$ & $r_{g}$ & $r_{h}$ & $r_{i}$ & $r_{f}$ & $r_{j}$ \\
\hline$R_{144^{\circ}}$ & $R_{144^{\circ}}$ & $R_{216^{\circ}}$ & $R_{288^{\circ}}$ & $I$ & $R_{72^{\circ}}$ & $r_{h}$ & $r_{i}$ & $r_{f}$ & $r_{j}$ & $r_{g}$ \\
\hline$R_{216^{\circ}}$ & $R_{216^{\circ}}$ & $R_{288^{\circ}}$ & $I$ & $R_{72^{\circ}}$ & $R_{144^{\circ}}$ & $r_{i}$ & $r_{f}$ & $r_{j}$ & $r_{g}$ & $r_{h}$ \\
\hline$R_{288^{\circ}}$ & $R_{288^{\circ}}$ & $I$ & $R_{72^{\circ}}$ & $R_{144^{\circ}}$ & $R_{216^{\circ}}$ & $r_{f}$ & $r_{j}$ & $r_{g}$ & $r_{h}$ & $r_{i}$ \\
\hline$r_{j}$ & $r_{j}$ & $r_{f}$ & $r_{i}$ & $r_{h}$ & $r_{g}$ & $I$ & $R_{288^{\circ}}$ & $R_{216^{\circ}}$ & $R_{144^{\circ}}$ & $R_{72^{\circ}}$ \\
\hline$r_{g}$ & $r_{g}$ & $r_{j}$ & $r_{f}$ & $r_{i}$ & $r_{h}$ & $R_{72^{\circ}}$ & $I$ & $R_{288^{\circ}}$ & $R_{216^{\circ}}$ & $R_{144^{\circ}}$ \\
\hline$r_{h}$ & $r_{h}$ & $r_{g}$ & $r_{j}$ & $r_{f}$ & $r_{i}$ & $R_{144^{\circ}}$ & $R_{72^{\circ}}$ & $I$ & $R_{288^{\circ}}$ & $R_{216^{\circ}}$ \\
\hline$r_{i}$ & $r_{i}$ & $r_{h}$ & $r_{g}$ & $r_{j}$ & $r_{f}$ & $R_{216^{\circ}}$ & $R_{144^{\circ}}$ & $R_{72^{\circ}}$ & $I$ & $R_{288^{\circ}}$ \\
\hline$r_{f}$ & $r_{f}$ & $r_{i}$ & $r_{h}$ & $r_{g}$ & $r_{j}$ & $R_{288^{\circ}}$ & $R_{216^{\circ}}$ & $R_{144^{\circ}}$ & $R_{72^{\circ}}$ & $I$ \\
\hline
\end{tabular}

Para $n \geq 3$, descreva os elementos de $D_{n}$. (Dica: você precisará considere dois casos - $n$ par e $n$ impar.) Quantos elementos tem $D_{n}$ ?

2. Em $D_{n}$, explique geometricamente por que uma reflexão seguida por uma reflexão deve ser uma rotação.

3. Em $D_{n}$, explique geometricamente por que uma rotação seguida por uma rotação deve ser uma rotação.

4. Em $D_{n}$, explique geometricamente por que uma rotação e uma reflexão tomadas juntas em qualquer ordem devem ser uma reflexão.

5. Associe o número $1 \mathrm{com}$ uma rotação e o número -1 com uma reflexão. Descreva uma analogia entre multiplicar esses dois números e multiplicar os elementos de $D_{n}$.

6. Se $r_{1}, r_{2}$ e $r_{3}$ representam rotações de $D_{n}$ e $f_{1}, f_{2}$ e $f_{3}$ representam reflexões de $D_{n}$, determine se $r_{1} r_{2} f_{1} r_{3} f_{2} f_{3} r_{3}$ é uma rotação ou uma reflexão. 
Grupos diedrais: uma proposta concreta para uma apresentação inicial da Álgebra Abstrata para licenciandos em Matemática

7. Suponha que $a, b$ e $c$ sejam elementos de um grupo diedral. É $a^{2} b^{4} a c^{5} a^{3} c$ uma rotação ou uma reflexão? Explique seu raciocínio.

8. Quais letras do alfabeto Inglês escritas em maiúsculas possuem um grupo de simetria com quatro elementos? Descreva as quatro simetrias.

9. Encontre os elementos $\mathrm{A}, \mathrm{B}$ e $\mathrm{C}$ em $\mathrm{D}_{4}$, de modo que $\mathrm{AB}=\mathrm{AC}$, mas $A \neq C$. (Assim, o "cancelamento cruzado" não é válido).

10. Explique o que o diagrama a seguir mostra sobre o grupo $\mathrm{D}_{\mathrm{n}}$.
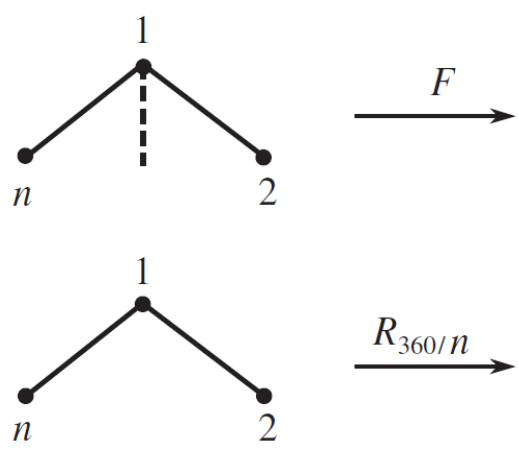
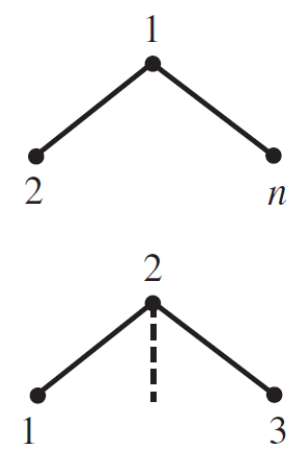
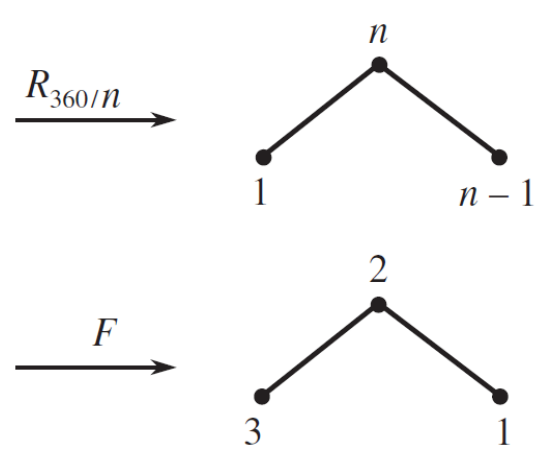

11. Descreva as simetrias de um retângulo não-quadrado. Construa a tabela Cayley correspondente.

12. Descreva as simetrias de um paralelogramo que não é nem um retângulo nem um losango. Descreva as simetrias de um losango que não seja um retângulo.

13. Descreva as simetrias de uma elipse não circular. Faça o mesmo com uma hipérbole. 
Grupos diedrais: uma proposta concreta para uma apresentação inicial da Álgebra Abstrata para licenciandos em Matemática

14. Considere uma faixa infinitamente longa de H's igualmente espaçados:

\section{... H H H ...}

15. Descreva as simetrias desta faixa (da questão anterior). O grupo de simetrias da faixa é Abeliano?

16. Para cada um dos flocos de neve, na figura a seguir, encontre o Grupo de simetria e localize os eixos de simetria reflexiva (desconsidere as imperfeições).

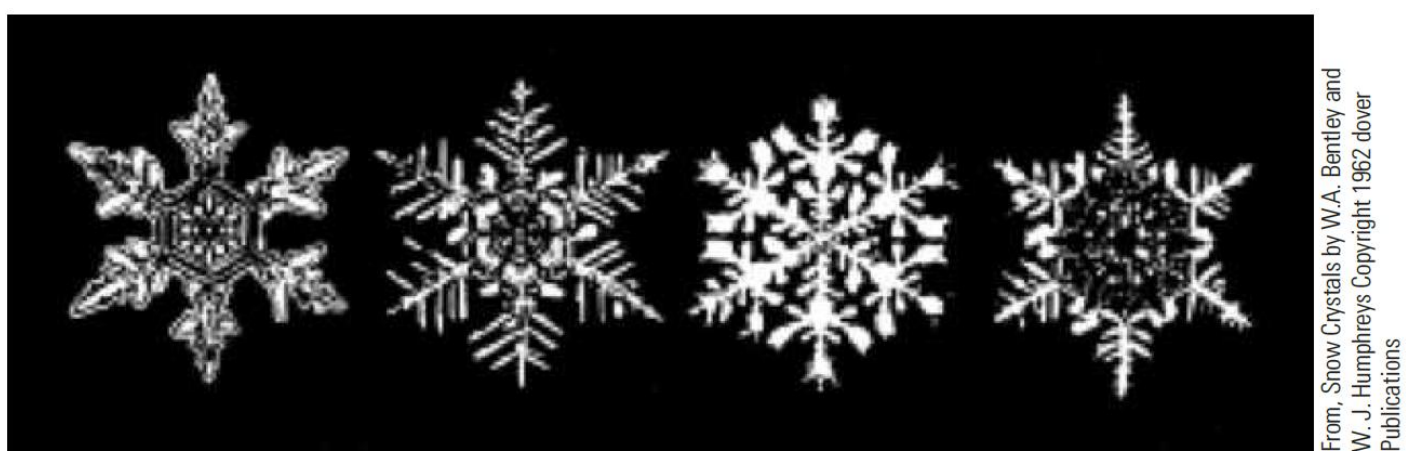

17. Determine o Grupo de simetria da camada externa da seção transversal do vírus da imunodeficiência humana (HIV) mostrado abaixo.

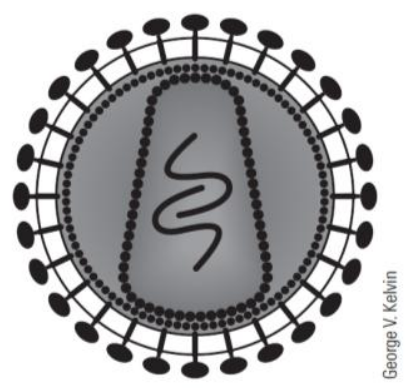

18. Sejam X, Y, R90 elementos de $D_{4}$ com $Y \neq R_{90}$ e $X^{2} Y=R_{90}$. Determine $Y$. Explique seu raciocínio. 
Grupos diedrais: uma proposta concreta para uma apresentação inicial da Álgebra Abstrata para licenciandos em Matemática

19. Se F é uma reflexão no grupo diedral $D_{n}$, encontre todos os elementos $\mathrm{X}$ em $\mathrm{D}_{\mathrm{n}}$ de forma que $\mathrm{X}^{2}=\mathrm{F}$ e todos os elementos $\mathrm{X}$ em $D_{n}$ tais que $X^{3}=F$.

20. Que propriedade de simetria as palavras em inglês "mow", "six" e "swims" têm quando escritas em letras maiúsculas?

21. Para cada logotipo abaixo, determine o Grupo de simetria (ignore as imperfeições).

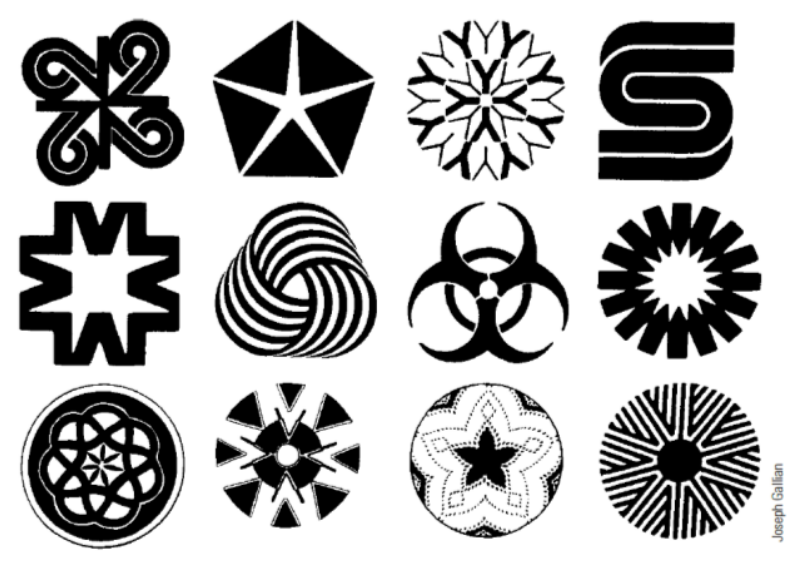

22. Que propriedade teórica de Grupo as letras maiúsculas F, G, J, $\mathrm{L}, \mathrm{P}, \mathrm{Q}$ e R têm que não são compartilhadas pelas letras maiúsculas restantes no alfabeto Inglês?

Artigo recebido em: 15 out. 2019 Artigo aprovado em: 20 nov. 2019 
Grupos diedrais: uma proposta concreta para uma apresentação inicial da Álgebra Abstrata para licenciandos em Matemática 